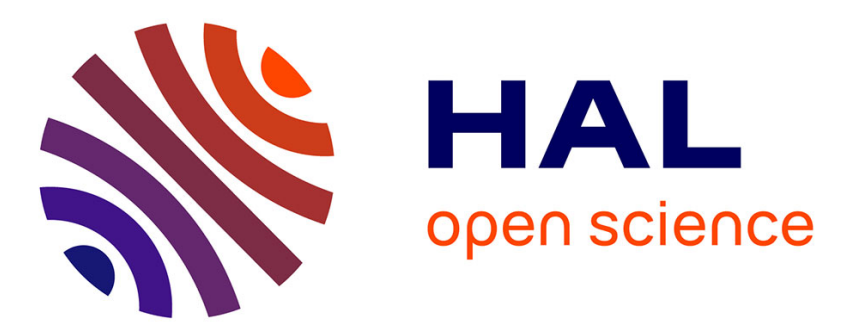

\title{
Etude de la propagation des ondes acoustiques dans les dièdres avec l'aide de la méthode des éléments finis
}

\author{
Anne-Christine Hladky, R. Bossut
}

\section{To cite this version:}

Anne-Christine Hladky, R. Bossut. Etude de la propagation des ondes acoustiques dans les dièdres avec l'aide de la méthode des éléments finis. Journal de Physique IV Proceedings, 1994, 04 (C5), pp.C5-689-C5-692. 10.1051/jp4:19945147 . jpa-00252825

\section{HAL Id: jpa-00252825 https://hal.science/jpa-00252825}

Submitted on 1 Jan 1994

HAL is a multi-disciplinary open access archive for the deposit and dissemination of scientific research documents, whether they are published or not. The documents may come from teaching and research institutions in France or abroad, or from public or private research centers.
L'archive ouverte pluridisciplinaire HAL, est destinée au dépôt et à la diffusion de documents scientifiques de niveau recherche, publiés ou non, émanant des établissements d'enseignement et de recherche français ou étrangers, des laboratoires publics ou privés. 


\title{
Etude de la propagation des ondes acoustiques dans les dièdres avec l'aide de la méthode des éléments finis
}

\author{
A.-C. HLADKY-HENNION et R. BOSSUT \\ IEMN, Département ISEN, 41 Boulevard Vauban, 59046 Lille cedex, France
}

\begin{abstract}
Résumé: Un modèle mathématique bidimensionnel a été développé pour étudier la propagation des ondes acoustiques dans les guides d'onde. Ce papier decrit d'abord l'approche theorique proposée puis présente l'application à un dièdre rectiligne élastique dont l'angle au sommet est variable. La comparaison à des résultats empiriques ou à des mesures permet la validation du modele. Ensuite, la méthode est étendue au cas des guides d'onde courbes.
\end{abstract}

\begin{abstract}
A bidimensional mathematical model has been developed to study the propagation of acoustic waves in waveguides. This paper presents the theoretical formulation and its application to a rectilinear elastic wedge, which top angle is variable. Comparison between finite element results and empirical or experimental results demonstrates the accuracy of the model. Then, the method is extended to curved waveguides.
\end{abstract}

\section{Introduction}

Depuis plusieurs années, un effort de recherche important a été accompli concernant les guides d'onde de surface et notamment les dièdres. En effet, dans ce type de guide, les modes de propagation sont peu dispersifs et leur vitesse de propagation est faible, inférieure à la vitesse des ondes de Rayleigh. De plus, l'énergie acoustique est confinée au sommet du diedre, qui seul subit des déformations importantes lorsque l'onde se propage. Ainsi, ces dièdres suscitent un grand intérêt, notamment dans les domaines du traitement du signal, pour la réalisation de lignes à retard ou de filtres acoustiques.

Les conditions aux limites de ce problème étant complexes, il est difficile d'utiliser un modèle simple pour y étudier la propagation des ondes. Ainsi S.L. Moss et al [1] ont établi un modèle analytique limité à des géométries simples de dièdres rectilignes. P.E. Lagasse [2] a décrit une technique numerique, basée sur la méthode des éléments finis, permettant de calculer les modes propres de guides d'onde rectilignes de section quelconque. La méthode utilisée est originale car elle réduit le problème du guide d'onde à un problème bidimensionnel, où seule la section du guide est maillée en éléments finis, tout en prenant en compte la propagation de l'onde dans la direction perpendiculaire au plan de la section (Fig. 1).

L'objet de cette communication est de présenter, dans une première partie, la méthode de Lagasse, implémentée dans le code éléments finis ATILA [3]. Plusieurs calculs sur des dièdres rectilignes en duraluminium dans l'air sont comparés, dans une deuxième partie, à des mesures [4] ou à des formules empiriques [2]. Enfin, la troisième partie présente une extension de la méthode au cas d'un dièdre courbe, formant par exemple un cône (Fig. 2). Dans ce cas, une méthode originale s'inspirant de la méthode de Lagasse permet de ne mailler que la section du cône, tout en prenant en compte la propagation des ondes autour du cône. Testée pour l'instant sur des exemples simples, la méthode développée donne de très bons résultats. 
Direction de

Propagation

(z)

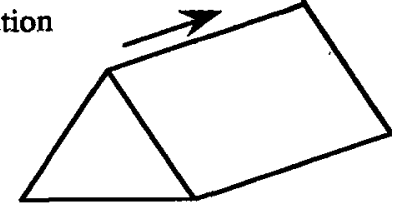

Dièdre élastique dans l'air.

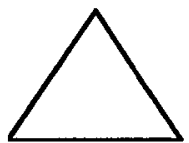

Section du dièdre faisant l'objet d'un maillage éléments finis.

Figure 1: Etude de la propagation des ondes acoustiques le long d'un dièdre

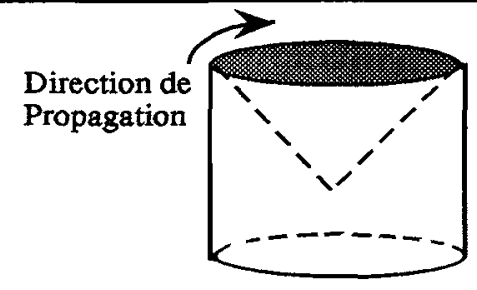

Cône élastique dans l'air.

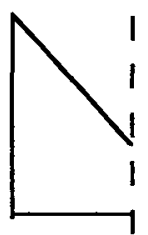

Section du cône faisant l'objet d'un maillage éléments finis.

Figure 2: Etude de la propagation des ondes acoustiques autour d'un cône

\section{Formulation théorique pour le guide d'onde rectiligne}

Une onde acoustique, caracterisée par son nombre d'onde $k$, se propage dans un guide d'onde, uniforme et infini suivant la direction $\mathrm{z}$ (Fig. 1). Le champ de déplacement peut s'écrire sous la forme:

$$
\mathrm{u}=\left[\begin{array}{c}
\mathbf{u}_{1}(\mathrm{x}, \mathrm{y}) \\
\mathbf{u}_{2}(\mathrm{x}, \mathrm{y}) \\
\mathrm{ju} \mathrm{u}_{3}(\mathrm{x}, \mathrm{y})
\end{array}\right] e^{\mathrm{j} \mathbf{k z}}
$$

où la dépendance temporelle en $\mathrm{e}^{-\mathrm{j} \omega t}$ est implicite dans les équations. Dans l'élément $\mathrm{e}$, le champ de déplacement peut s'écrire en fonction du vecteur des valeurs nodales du champ de déplacement $\underline{U}^{e}$ :

$$
\mathbf{u}=\left[\mathbf{N}^{\mathrm{e}}\right] \mathbf{U}^{\mathrm{e}}
$$

où la matrice $\left[\mathrm{N}^{\mathrm{e}}\right]$ correspond aux fonctions d'interpolation associées à l'élément. L'écriture du champ de deplacement sous la forme de l'équation (1) modifie l'expression du tenseur des déformations $\mathbf{S}$ [3]. Dans les systèmes éléments finis classiques, seule la matrice $\left[\mathrm{B}^{\mathrm{e}}\right]$, matrice des dérivées spatiales des fonctions d'interpolation reliant le vecteur du champ de déplacement et le tenseur des déformations dans l'élément e, est modifiée et a pour expression :

$$
\left[\mathbf{B}^{\mathrm{e}]}=\left[\begin{array}{cccccc}
\frac{\partial\left[\mathrm{N}^{\mathrm{e}}\right]}{\partial \mathrm{x}} & 0 & 0 & 0 & j k\left[\mathrm{~N}^{\mathrm{e}}\right] & \frac{\partial\left[\mathrm{N}^{\mathrm{e}}\right]}{\partial \mathrm{y}} \\
0 & \frac{\partial\left[\mathrm{N}^{\mathrm{e}}\right]}{\partial \mathrm{y}} & 0 & j k\left[\mathrm{~N}^{\mathrm{e}}\right] & 0 & \frac{\partial\left[\mathrm{N}^{\mathrm{e}}\right]}{\partial \mathrm{x}} \\
0 & 0 & -k\left[\mathrm{~N}^{\mathrm{e}}\right] & \frac{\partial\left[\mathrm{N}^{\mathrm{e}}\right]}{\partial \mathrm{y}} & \frac{\partial\left[\mathrm{N}^{\mathrm{e}}\right]}{\partial \mathrm{x}} & 0
\end{array}\right]^{T}\right.
$$

Cette matrice intervient dans le calcul de la matrice élémentaire de rigidité mécanique $\left[\mathrm{K}_{\mathrm{uu}}^{\mathrm{e}}\right]$, definie par la relation: 


$$
\left[K_{u u}^{e}\right]=\int_{\Sigma_{e}}\left[B^{e}\right]^{* T}[C]\left[B^{e}\right] d \Sigma_{e}
$$

où [c] est le tenseur de rigidité et $\Sigma_{\mathrm{e}}$ la surface de l'élément e. Compte tenu de l'expression de la matrice $\left[\mathrm{B}^{\mathrm{e}}\right]$, la matrice $\left[\mathrm{K}_{\mathrm{uu}}^{\mathrm{e}}\right]$ peut s'écrire sous la forme:

$$
\left[\mathbf{K}_{u v}^{\mathrm{e}}\right]=\left[\mathbf{K}_{0}^{\mathrm{e}}\right]+\mathbf{k}\left[\mathrm{K}_{1}^{\mathrm{e}}\right]+\mathrm{k}^{2}\left[\mathrm{~K}_{2}^{\mathrm{e}}\right]
$$

où les matrices $\left[\mathrm{K}_{0}^{\mathrm{e}}\right],\left[\mathrm{K}_{1}^{\mathrm{e}}\right]$ et $\left[\mathrm{K}_{2}^{\mathrm{e}}\right]$ sont réelles symétriques. Pour chaque valeur du nombre d'onde $\mathrm{k}$, la matrice de rigidité totale $\left[\mathrm{K}_{\text {uu }}\right]$ résulte de l'assemblage des matrices de rigidité élementaires $\left[\mathrm{K}_{\text {uud }}^{\mathrm{e}}\right.$. Classiquement, le système à résoudre reste [3] :

$$
\left(\left[\mathrm{K}_{\mathrm{uu}}\right]-\omega^{2}[\mathrm{M}]\right) \mathrm{U}=0
$$

La résolution du système donne les pulsations propres $\omega$ de la structure pour un nombre d'onde $\mathrm{k}$ donné et le vecteur $\underset{U}{\mathrm{U}}=\left(\mathrm{U}_{1}, \mathrm{U}_{2}, \mathrm{U}_{3}\right)^{\mathrm{T}}$ associé. Les courbes de dispersion sont obtenues en traçant l'évolution des pulsations propres en fonction du nombre d'onde. Connaissant le champ de déplacement dans le plan $z=0$ par l'equation (1): $\left(\mathrm{U}_{1}, \mathrm{U}_{2}, \mathrm{j}_{3}\right)^{\mathrm{T}}$ il est possible de reconstituer le champ de déplacement du guide d'onde entier en multipliant ce vecteur par $\mathrm{e}^{\mathrm{jkz}}$.

\section{Résultats}

Le dièdre rectiligne étudié dans cette partie a un angle au sommet variable. Le materiau est le duraluminium, dont les caractéristiques sont $c_{L}=6350 \mathrm{~ms}^{-1}, c_{T}=3100 \mathrm{~ms}^{-1}$ et $\rho=2700 \mathrm{~kg} \cdot \mathrm{m}^{-3}$. La vitesse $c_{R}$ des ondes de Rayleigh est égale à $2975 \mathrm{~ms}^{-1}$. Dans le but d'étudier les ondes localisées au sommet du dièdre, ondes de flexion antisymétriques par rapport au plan médian du dièdre, seule la moitié de la structure est maillée par éléments finis, en imposant une condition d'antisymétrie. Lorsque la longueur d'onde est petite, la hauteur $h$ du dièdre maillé a peu d'influence sur les résultats puisque l'onde de dièdre est localisée au sommet. Il faut cependant que le maillage soit très fin au voisinage de la pointe. La figure 3 présente les variations des vitesses de propagation des premières ondes du dièdre, normalisées à la vitesse des ondes de Rayleigh, en fonction de l'angle au sommet. Ces vitesses sont les vitesses de phase $(\omega / k)$ déterminées à partir des courbes de dispersion. La figure 3 présente de même les résultats expérimentaux [4] et les résultats de la formule empirique de Lagasse [2]: $c=c_{R} \sin (m \alpha)$, $m$ entier tel que $\mathrm{m} \alpha<90^{\circ}$, où $\alpha$ est l'angle au sommet du diedre. L'accord entre les courbes est correct. La figure 3 montre la limite de la formule empirique, notamment lorsque l'angle au sommet du dièdre est grand. Ces résultats sont très proches des résultats obtenus par S.L.Moss [3]. La figure 4 présente le champ de déplacement typique du premier mode de diedre, lorsque l'angle au sommet est de $30^{\circ}$, et montre que l'onde est localisée au sommet du dièdre.
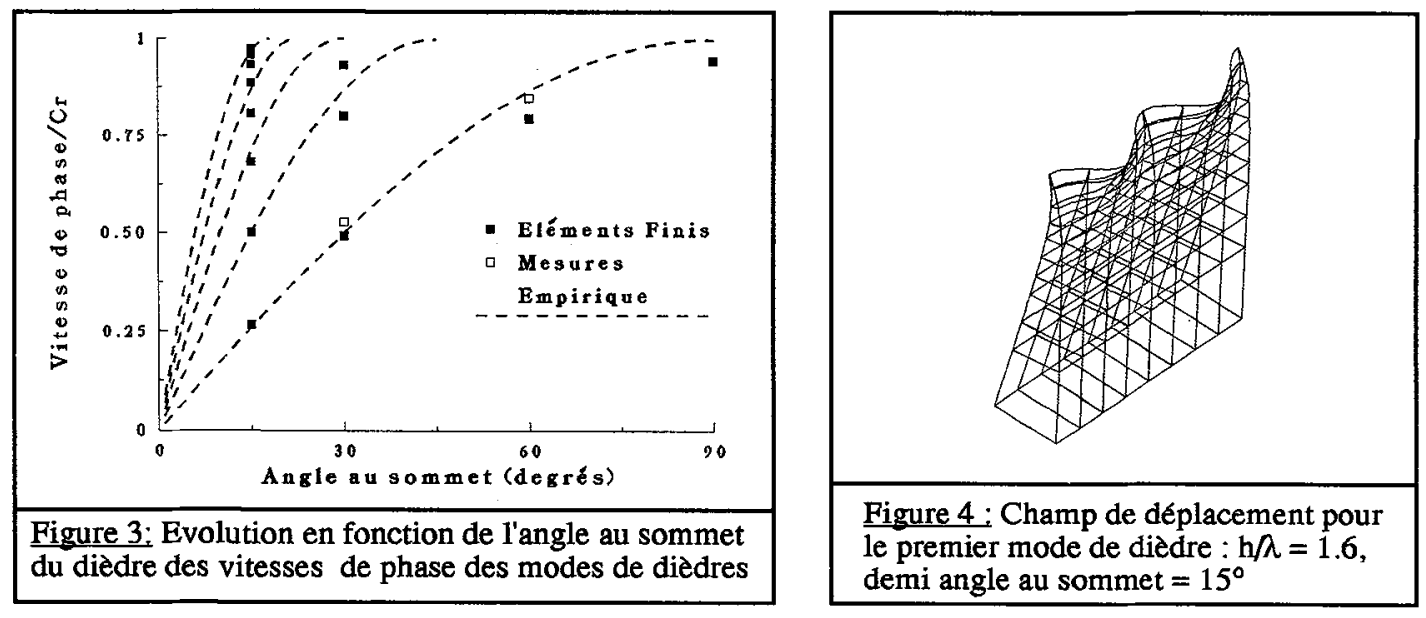


\section{Formulation théorique pour le guide d'onde courbe}

Cette partie présente l'extension de la méthode au cas d'un guide d'onde courbe, de rayon de courbure constant, formant par exemple un cône (Fig. 2). Dans ce cas, une onde acoustique, caractérisée par son nombre d'onde $\mathrm{k}$, se propage dans le guide d'onde courbe et le champ de déplacement u peut s'écrire sous la forme:

$$
\mathrm{u}=\left[\begin{array}{c}
\mathrm{u}_{1}(\mathrm{x}, \mathrm{y}) \\
\mathrm{u}_{2}(\mathrm{x}, \mathrm{y}) \\
\mathrm{ju} \mathrm{u}_{3}(\mathrm{x}, \mathrm{y})
\end{array}\right] \mathrm{e}^{\mathrm{jk \theta} \theta}
$$

où $x$ et $y$ correspondent au plan de la section du cône et $\theta$ varie de 0 a $2 \pi$ pour couvrir tout le cône. Le nombre d'onde $k$ est un entier, puisque le régime est supposé établi. Seule la matrice $\left[\mathrm{B}^{\mathrm{e}}\right]$ est modifiée et a pour expression :

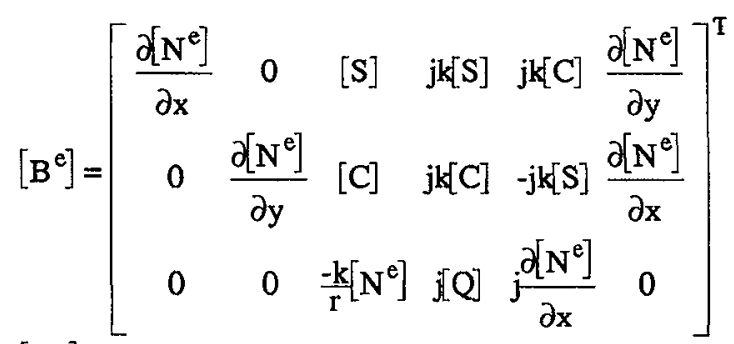

avec $:[Q]=\frac{-1}{r}\left[N^{e}\right]+\frac{\partial\left[N^{e}\right]}{\partial y},[S]=\frac{\sin \alpha}{r}\left[N^{e}\right]$ et $[C]=\frac{\cos \alpha}{r}\left[N^{e}\right]$. $r$ est la distance entre le point consideré et l'axe de symétrie, $\alpha$ est l'angle entre l'axe $\mathrm{x}$ global et l'axe $\mathrm{x}$ local [3]. Cette matrice intervient dans le calcul de la matrice élémentaire de rigidité mécanique $\left[\mathrm{K}_{\mathrm{uu}}^{\mathrm{e}}\right]$, définie par la relation (4). Compte tenu de l'expression de la matrice $\left[\mathrm{B}^{\mathrm{e}}\right]$, la matrice $\left[\mathrm{K}_{\text {uu }}^{\mathrm{e}}\right]$ peut s'écrire sous la forme de l'equation (5) où les matrices $\left[\mathbf{K}_{0}^{\mathrm{e}}\right],\left[\mathbf{K}_{1}^{\mathrm{e}}\right]$ et $\left[\mathbf{K}_{2}^{\mathrm{e}}\right]$ sont une nouvelle fois réelles symétriques. La résolution du système classique (6) donne les pulsations propres $(n$ de la structure pour un nombre d'onde $\mathrm{k}$ donné et le vecteur $\mathrm{U}=\left(\mathrm{U}_{1}, \mathrm{U}_{2}, \mathrm{U}_{3}\right)^{\mathrm{T}}$ associé. Connaissant le champ de déplacement dans le plan $\theta=0$ par l'équation (7) : $\left(\mathrm{U}_{1}, \mathrm{U}_{2}, \mathrm{U}_{3}\right)^{\mathrm{T}}$ il est possible de reconstituer le champ de déplacement du guide d'onde courbe entier en multipliant ce vecteur par $\mathrm{e}^{\mathrm{jk} \theta}$. Testée dans le cas d'un anneau d'une part maillé entièrement et d'autre part lorsque seule la section de l'anneau est maillée, la méthode développée donne de très bons résultats.

\section{Conclusion}

L'analyse de la propagation des ondes acoustiques dans un guide d'onde rectiligne ou courbe est conduite avec l'aide du code éléments finis ATILA. Un bon accord est trouvé entre les résultats numériques et les mesures. Les limites d'un modèle empirique sont de plus soulignées. L'un de nos objectifs est maintenant d'étudier plusieurs types de guides d'onde courbes. De même, il est envisagé de prendre en compte l'effet du fluide extérieur dans le cas où les guides d'onde sont immergés.

\section{Références}

[1]. S.L. Moss, A.A. Maradudin, S.L. Cunningham, "Vibrational edge modes for wedges with arbitrary interior angles", Phys. Rev., $\underline{8}, 2999-3008,(1973)$.

[2]. P.E. Lagasse, "Higher-order finite-element analysis of topographic guides supporting elastic surface waves", J. Acoust. Soc. Am., 53, 1116-1122, (1973).

[3]. J.-N. Decarpigny, "Application de la méthode des éléments finis à l'étude de transducteurs piézoélectriques", Thèse de Doctorat ès Sciences Physiques, Université des Sciences et Techniques de Lille, (1984).

[4]. X. Jia, M. De Billy, "Observation of the dispersion behavior of surface acoustic waves in a wedge waveguide by laser-ultrasonics", Applied Physic Letters, 61, 2970-2972, (1992). 